\title{
Power Flow Controller for The Meshed DC Grids
}

\author{
Chiheng Gong ${ }^{1, a}$, Shujnu Yao ${ }^{2, b}$ and Haiyang Guo ${ }^{2, c}$ \\ ${ }^{1}$ School of North China Electric Power University University, Beijing 102206, China; \\ ${ }^{2}$ School of North China Electric Power University University, Beijing 102206, China; \\ a18811169672@163.com, bopen2000@sina.com, 'bj_leaf@126.com
}

Keywords: Renewable energy, Meshed DC grids, Power flow control devices

\begin{abstract}
With more and more environmental problems, renewable energy begins to play an increasingly large proportion. High-voltage direct current technology based on voltage source converter (VSC-HVDC) enables smooth access to renewable energy. Traditional DC grids system is generally radial connections, but In recent years, there are many parallel lines between the two converter stations in some meshed DC grids. However, the converter stations can't control the power flow of parallel lines completely, and some lines might cause too much loss even overload.According to the theory of circuit,there are four types of power flow control devices, variable resistors, DC transformers, CFC and series voltage sources have been proposed for the power flow control in a meshed DC grid .In this paper, their topology and operation properties were compared.
\end{abstract}

\section{Introduction}

New Energy is part of the renewable energy sources, their common features are: low energy density, hidden dispersion, Intermittent, random. Therefore their development and use subject to certain restrictions, technically there is a certain degree of difficulty.[4]With a variety of large-scale renewable energy connected to the grid, the traditional power equipment, power grid construction and operation technologies in receiving ultra large-scale renewable energy increasingly powerless, this must be the introduction of new technology, new equipment and the new network structure to meet the profound changes in the future energy landscape. HVDC technology based on voltage source converter (VSC) is an effective method which can solve the renewable energy parallel in grid, asynchronous interconnection of AC grid, energy emergency support and other issues. [5]

The three basic topology of DC grid are dendritic, ring, mesh network, and various combinations of the basic structure generate the complex DC Grid. Among them, the meshed network structure has better flexibility and redundancy to increase the reliability of the system and reduce the transmission distance, which is a firstly considered structure for the DC power grid construction in the future.[6]-[8] In recent years, in order to meet the DC grid development needs, many complex grid appears in the form of a few parallel lines between the two converter stations. [9] In this case, the converter station can not control the power flow of the DC lines entirely by adjusting the terminal voltage and current, because the current of the line is determined by the resistance of the line to some extent. [10] Thus, power flow control device for DC Grid is necessary, not only to achieve economic operation of the grid, but also to prevent the circuit of overload run in the fault condition.

\section{Comparative study of various kinds of Power Flow Controller}

There are mainly four DC power flow control equipment researched at home and abroad, namely variable resistor, DC transformer, current flow controller and series voltage source. [5],[11]-[15]The following are brief introductions of those four kinds of controllers, and compare their advantages and disadvantages.

\section{Variable Series Resistor}

We know that the DC current flowing through the DC grid is determined by the line resistance, so even a very small change can have a significant effect. Therefore, the DC current of the grid can be 
controlled by simply adding additional resistance to the line of least resistance. Reference [11] gives a basic structure of variable series resistor, as shown in FIG.1 In this method, each resistor switching into the DC line to complete the line current regulation, in order to achieve the effect of power flow control.

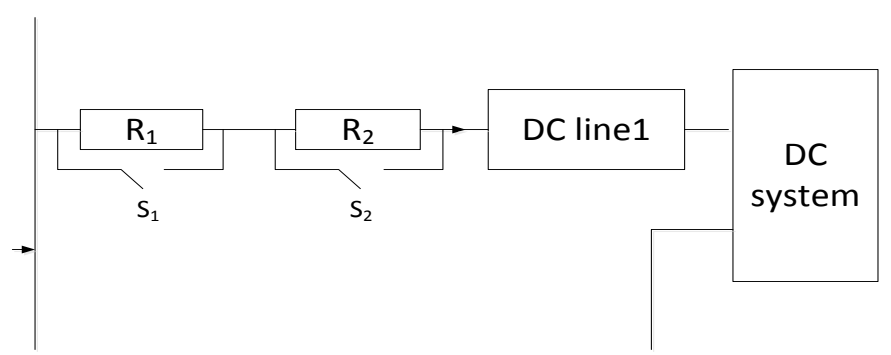

Fig. 1 Variable Series Resistor control circuit current

Reference [5] gives another detailed topology, as shown in Fig.2.This topology consists of two anti-parallel resistors connected in parallel to the positive and negative poles. The meaning of the anti-parallel resistors is to keep the balance of positive and negative transmission effects from the device. P1, P2 access to positive electrode, and N1,N2 access to negative electrode.The inductor series in the line is for smoothing and mitigate the impact of switching equipment. Bi-directional IGBT can achieve two-way power flow. The diodes connected with in series is to withstand the reverse voltage, because the IGBT can hardly withstand the reverse voltage.

The equivalent resistance accessed can be controlled by the control of the switch, the principle formula is as follows:

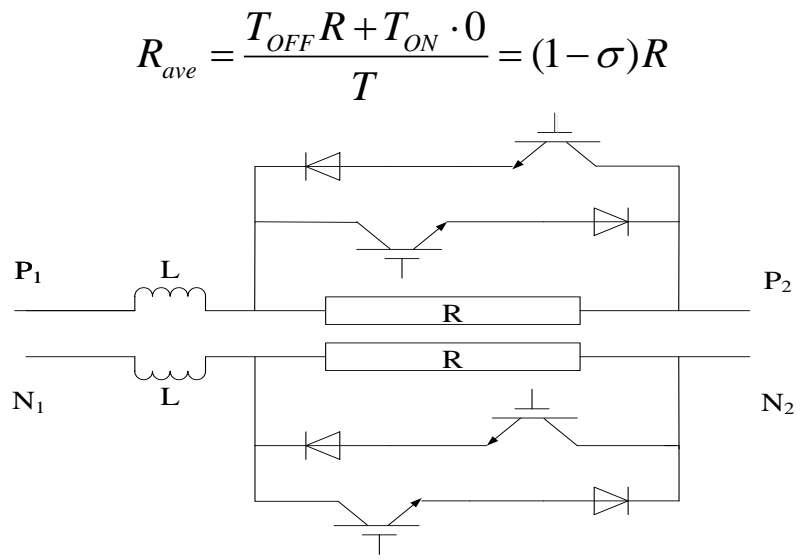

Fig. 2 Topology of the Variable Series Resistor

Under normal circumstances, it depends on several factors when the device can be applied in the power system. One is that the IGBT of the devices suitable for the trend reversal of the VSC. The second is the average resistance value of the resistor can be a smooth change to the reference value. Because of its simple structure, it can easily access to an existing project. But the series resistance consume additional power loss, so it is not suitable for the economic operation.

\section{Transformer}

DC transformer not only can be used to connect the DC grid with different voltage levels, but can also be used in the grid with the same voltage level. Reference [11] proposed a general model of a DC transformer, as shown in FIG.3.In this model, the primary side is current source $\mathrm{I}_{\mathrm{SR}}$, paralleled with a capacitor $C_{E}$; The secondary-side is voltage source $U_{S R}$, and series with the equivalent inductance $L_{E}$. $\mathrm{L}_{1}$ And $\mathrm{C}_{2}$ as the real component of the DC transformer is also included. The output voltage of the secondary side $\mathrm{U}_{\mathrm{SR}}$ can be individually controlled. According to the principles of the transformer, $\mathrm{I}_{\mathrm{SR}}$ can be calculated according to the formula as follows:

$$
I_{S R}=\frac{U_{S R} I_{d 2}}{U_{d 1}}
$$




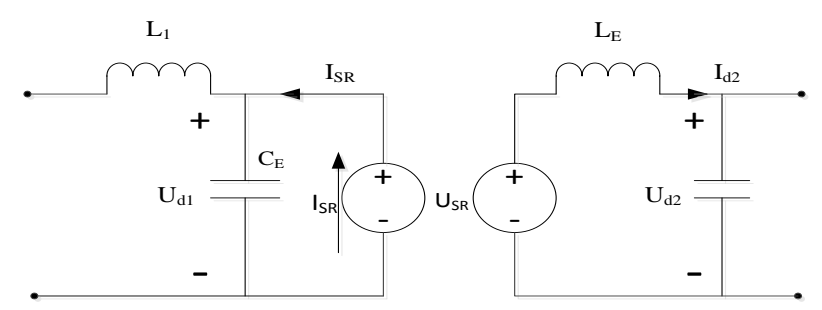

Fig. 3 General Model of DC transformer

In order to achieve complete control of the injected electrical energy, DC transformer will adjust the output voltage. Control method of DC transformer's power flow is shown in Fig.4,which is composed of two parts. The first part is power flow regulation. There are two input signals, the expected current of DC line $I_{d r e f}$ and the actual current $I_{d}$ Thereafter, the error current signal is sent to a PID controller. Another part is used to generate the reference voltage, which uses the received signal adjustment and generating the reference voltage of $\mathrm{U}_{\mathrm{SR}}$. In this session, $\mathrm{U}_{0}$ is the rated voltage of the DC grid, so as to ensure the normal operation of the DC transformer.

Due to the voltage of DC grid has no uniform standard, there is various voltage levels of the DC grid, so the DC transformer is essential equipment. So far the DC converter has no application in the DC transmission, but there will be a lot of DC transformer applications in the future. Ideally, DC transformer needs to achieve the following functions: high transformation ratio, controllable, ladder-type ratio, to connect different voltage levels DC system; can be connected to different types of inverter; power balance between DC system poles; controllable bidirectional flow direction; low loss, low cost, small size; has a certain capability of current fault withstand .[12]

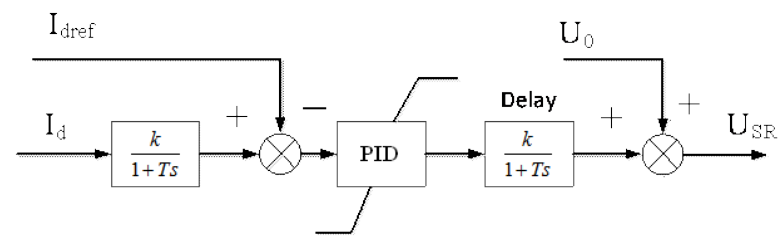

Fig. 4 Power flow control diagram of DC transformer

\section{Current Flow Controller ( CFC)}

Reference [5] proposed two topology of direct current power flow controller (CFC), one is shown in Figure 5. The two converter modules in this topology are connected in series with two DC lines. The voltage of each converter module is formed from pulse width modulation by the power switching devices (typically IGBT), to control the current flow in the conductor. Since the device is actually only play a role in the rational allocation of direct current, so there is no power loss in theory. In practice, however, there will still be a corresponding power switching and conduction losses.

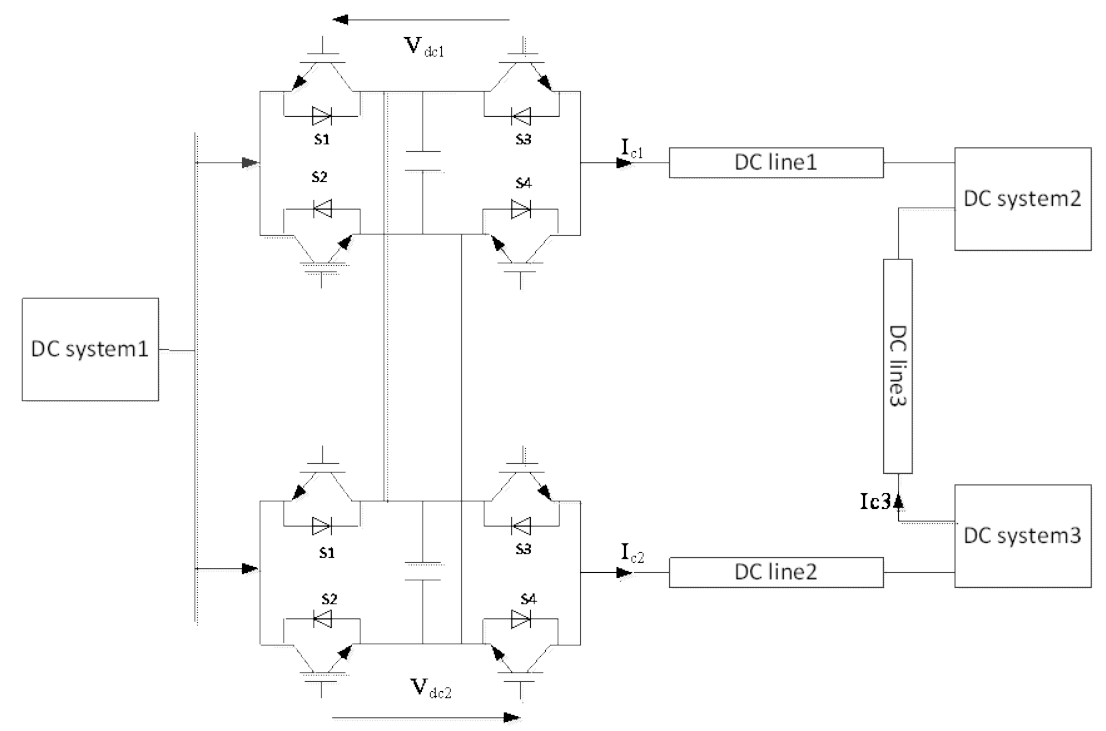

Fig. 5 Current flow controller model one 
Reference [13] researched another topology of CFC proposed in reference [5].Figure 6 shows this kind of CFC applied in the DC grid.

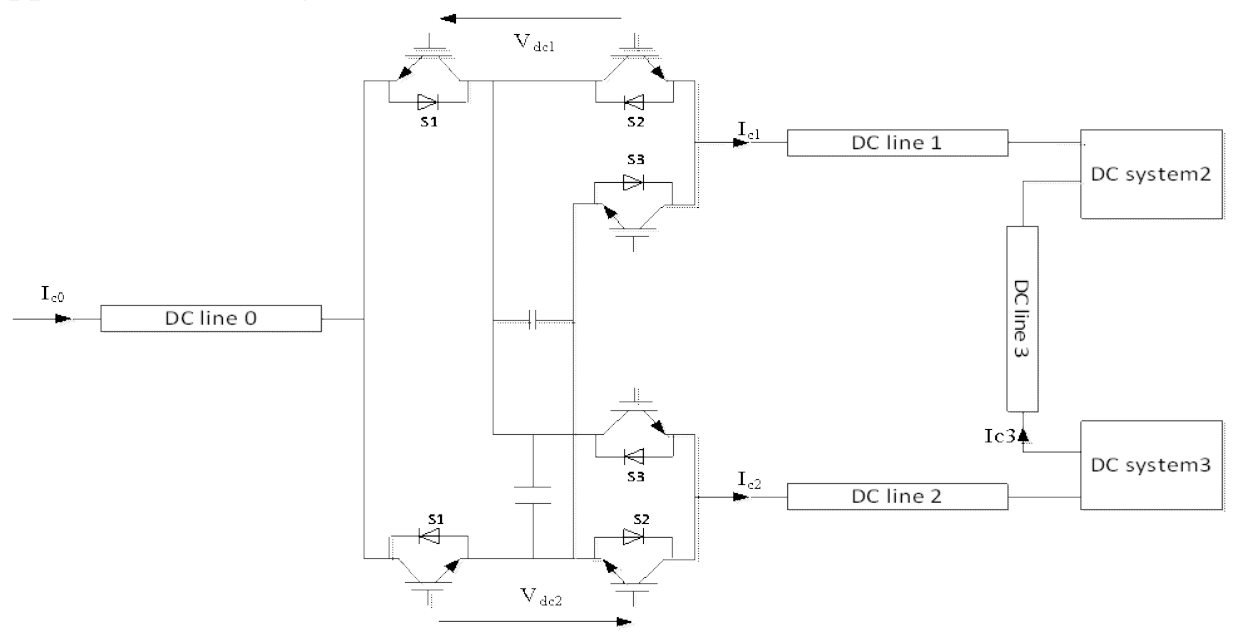

Fig. 6 Current flow controller model two

CFC can take energy from other DC branch point, so the power electronic devices don't need to withstand the high DC voltage, nor need a converter transformer. DC current is very sensitive to the change of DC voltage, and the average voltage on branch 1 and 2 introduced by the CFC is much smaller compared to the DC voltage. The capacitor voltage is also smaller, and the voltage which power electronic devices in the CFC have to withstand is closely related to the capacitor voltage, so the CFC required less number of power electronics. Thus, the investment of CFC is less. Meanwhile, the less number of electronic devices means low running loss, which is favorable to the long-term economic operation. However, there are numbers of working conditions for CFC, and the control strategy is much more complex, so is it suitable for the practical project remains to be seen.

\section{Series Voltage Source}

Reference [5] [11] [14] [15] have proposed an series voltage source concept, and include the detail topology and control methods. The principle is similar, which is taking the energy from AC system into DC voltage source through rectifier and connect in series with the DC line, enabling power flow control of the DC grid. This method is actually replacing the series resistance of a voltage source, so as to achieve better control. The form of series voltage source access into the DC grid shown in Figure 7.

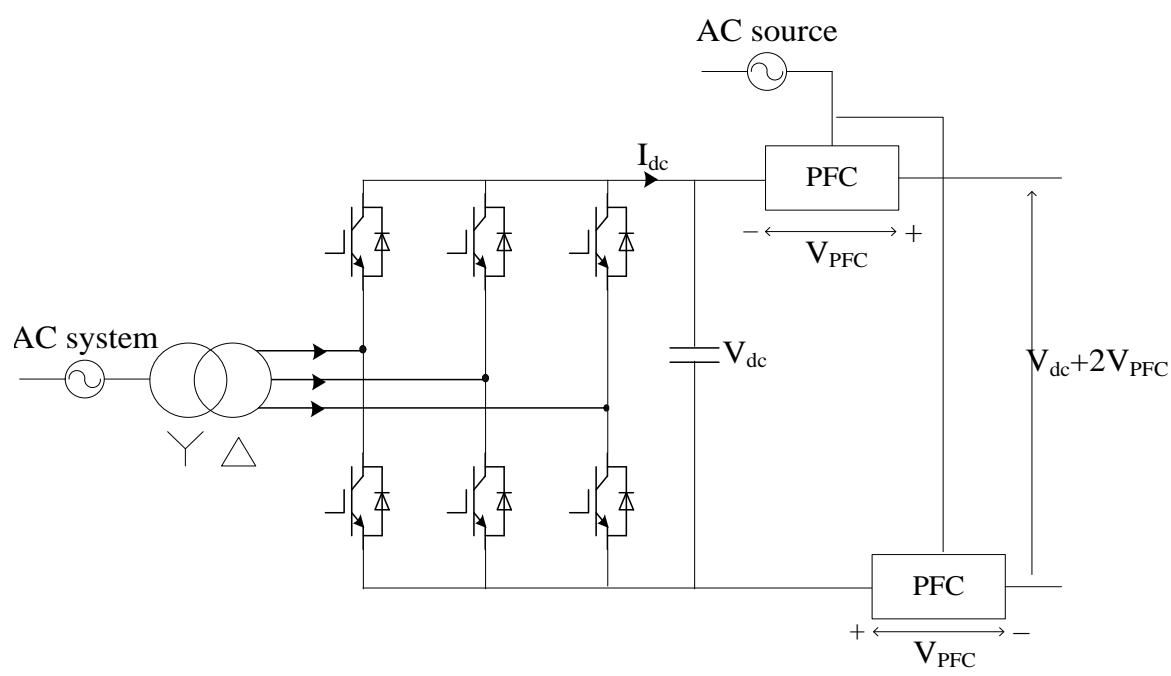

Fig. 7 Series voltage source access in the DC grid

Figure 8 shows the specific topology of the series voltage source. Similar to the above-mentioned second series resistor model, this model also includes two identical modules to balance the positive electrode and the negative electrode lines.AC power is connected to the AC side of the converter via a transformer, which is used to isolate the $\mathrm{AC}$ and DC power grids, and can also reduce the current injected into the AC system. AC/DC converter using a typical 6-pulse voltage source converter to 
maintain the voltage of the capacitor. DC/DC converter is used to adjust the inverter's output voltage so that the voltage accessed to the DC grid will finally meet the power flow control requirements. The DC/DC converter a four-quadrant chopper, as shown in Fig.8.

The control system of the device shown in Figure 9, wherein the PWM generator is prevalent in the actual project. The control system includes power flow regulator and a reference voltage generator. Two input of the regulator are the reference current $\mathrm{I}_{\mathrm{dref}}$ and the actual current $\mathrm{I}_{\mathrm{d}}$. Then generates a reference voltage $\mathrm{V}_{\mathrm{PFCref}}$ finally into the PWM module, and the DC/DC converter will be adjusted quickly.

It is worth mentioning that there usually has single-phase or three-phase AC power system and transformer nearby the converter station, so the device can be easily implemented in the practical project

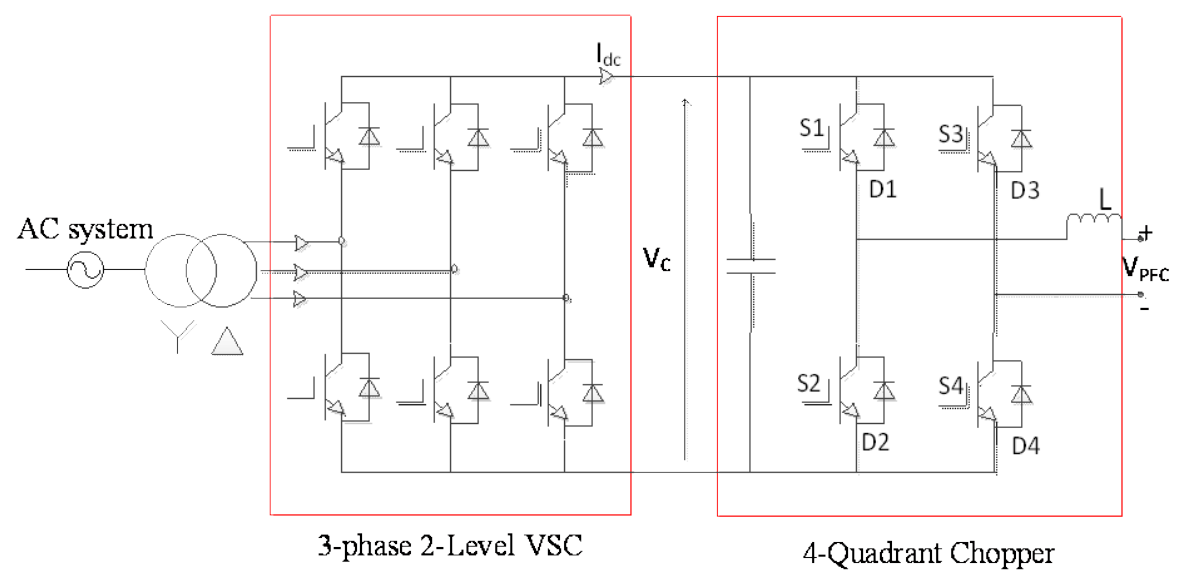

Fig.8 Topology of the series voltage source

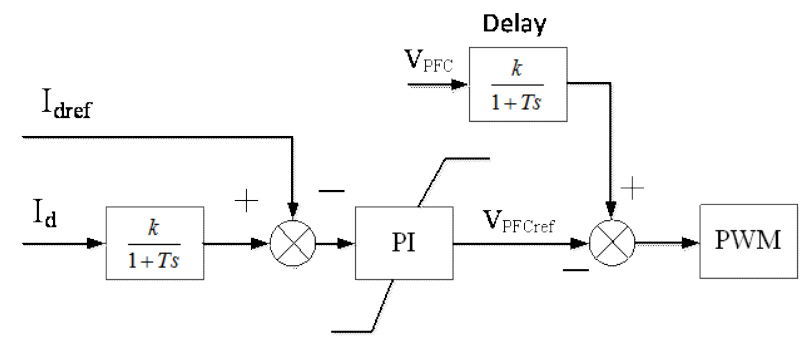

Fig.9 The control method of the series voltage source

\section{Conclusion}

Four types of power flow control devices, variable resistors, DC transformers, CFC and series voltage sources have been proposed for the power flow control in a meshed DC grid. The topology of the devices were designed. Each kind of devices has their advantages and disadvantages. Comparatively, the series voltage sources is the best choice for the power flow controller, and farther research will be done to verify the feasibility of the Series Voltage Source.

\section{Reference}

[1] Zhang Weibo, Pan Yuchao, Cui Zhiqiang. Development Approach of China's New Energy Power Generation [J].Energy of China,2012.

[2] Li Sheng, Li Yutang, Li Junling. New Energy Review[J].Modern Agriculture.2013.

[3] Gao Ze; Yang Jianhua; Feng Yuqing; Wang Yansong; Jin Feng. New Energy-based Power Generation Today and Analysis[J].SINO-GLOBAL ENERGY,2014.

[4] Xiao Wei. Analysis of New Energy Generation Status and Trends [J]. China Urban Economy, 2011. 
[5] Mu Q, Liang J, Li Y L, et al.Power flow control devices in DC grids[C]//2012 IEEE Power and Energy Society General Meeting.San Diego, CA: IEEE, 2012:1-7.

[6] Bucher M K, Wiget R, Andersson G, et al. Multi-terminal HVDC networks--what is the preferred topology?[J].IEEE Transactions on Power Delivery,2014,29(1):406-413.

[7] Wen Jialiang, Wu Rui, Peng Chang,et al.Analysis of DC grid prospects in China[J].Proceedings of the CSEE,2012,32(13):7-12(in Chinese).

[8] Tang Guangfu. A review of 2004 CIGRE on application status and perspective in HVDC and power electronics[J].Automation of Electric Power Systems,2005,29(7):1-5(in Chinese).

[9] Juhlin L E. Power flow control in a meshed HVDC power transmission network[P].US 20120033462Al,2011.

[10] Xiaobo Hu,Jun Liang,Rogers,D.J.Yalou Li.Power Flow and Power Reduction Control Using Variable Frequency of Offshore AC Grids[J].Power SystemsIEEE Transactions on(Volume:28Issue: 4),2013,3897 - 3905.

[11] Barker C D,Whitehouse R S.A current flow controller for use in HVDC grids[C]//10th IET International Conference on AC and DC Power Transmission(ACDC 2012).Birmingham,UK:IET, 2012:1-5.

[12] Tang Guangfu, Luo Xiang, Wei Xiaoguang, Multi-terminal HVDC and DC-grid technology [J].Proceedings of the CSEE,2013,33(10):8-17(in Chinese).

[13] XU Feng, XU Zheng, LIU Gaoren. A Neotype of DC Power Flow Controller and Its Applications in Meshed DC Grids[J].Power System Technology,2014,38(10).

[14] Balasubramaniam,S.Jun Liang,Ugalde-Loo,C.E.An IGBT Based Series Power Flow Controller for Multi-Terminal HVDC Transmission[C]//Power Engineering Conference (UPEC),2014 49th International Universities,IEEE,2014,1-6.

[15] Tianqi Zhang,Chuanyue Li,Jun Liang.A Thyristor Based Series Power Flow Control Device for Multi-Terminal HVDC Transmission.[C]//Power Engineering Conference (UPEC),2014 49th International Universities,IEEE,2014,1-6. 